Article

\title{
Demodulation of Angular Position and Velocity from Resolver Signals via Chebyshev Filter-Based Type III Phase Locked Loop
}

\author{
Huan Liu@ and Zhong Wu *(i) \\ School of Instrumentation and Optoelectronic Engineering, Beihang University, Beijing 100191, China; \\ liuhuan@buaa.edu.cn \\ * Correspondence: wuzhong@buaa.edu.cn; Tel.: +86-10-8233-9703
}

Received: 26 October 2018; Accepted: 21 November 2018; Published: 25 November 2018

\begin{abstract}
A high-accuracy demodulation algorithm is required to estimate angular position and angular velocity from resolver signals. In order to improve the estimation accuracy of conventional phase-locked loop (PLL) based demodulation method, a Chebyshev filter-based type III PLL method is proposed in this paper. The proposed method makes PLL become a system of type III tracking loop, which could greatly reduce the theoretical constant deviation in the estimation results of conventional type II PLL in case of variable speed. Meanwhile, the eigenvalues of type III PLL are placed to be the same position as those of a Chebyshev low-pass filter. In this way, demodulation parameters with stronger filter properties can be obtained to effectively suppress the high-frequency measurement noise in resolver signals. Thus, the proposed method can achieve higher demodulation precision compared with the conventional ones. Simulations and experiments are performed to validate the proposed demodulation method.
\end{abstract}

Keywords: demodulation; phase-locked loop; Chebyshev filter; measurement noise suppression

\section{Introduction}

Modern control algorithms for servomotors require accurate feedback information of both angular position and angular velocity. Usually, the angular position and velocity can be measured by shaft sensors, such as optical encoders, magnetic encoders, and resolvers. Compared with other sensors, resolvers have gained more attention owing to their various advantages such as simple and rugged structure, low cost, high accuracy, resistance to harsh environments [1-3]. Normally, a resolver generates two amplitude-modulated analog signals with rotor position information. Therefore, high-accuracy resolver-to-digital conversion (RDC) is required in order to extract rotor position and velocity from the resolver signals [4].

The special integrated circuit (IC)—such as AD2S80 series, AD2S83, AD2S1210 and PGA411-Q1— can conduct the RDC process to obtain angular position and velocity, and they are quite convenient to use. These commercial RDC ICs are mainly based upon type-II tracking loop technique, can continuously track the inputs and convert the input sine and cosine signals into a digital representation of rotor position and velocity. However, the cost of the RDC ICs is very high, even higher than the resolver itself; meanwhile, the typical RDC ICs have a limited bandwidth of 300-600 Hz [5], and it is difficult to adjust the parameters of RDC ICs to meet users' specific requirements.

In order to reduce costs and facilitate bandwidth adjustment of RDC, software-based RDC can be adopted [6,7]. To reduce the sampling pressure of CPU, generally, it is necessary to pre-detect the sine and cosine envelope of resolver outputs, and then demodulate the envelope signals to derive rotor position and velocity. 
The commonly used demodulation methods may be divided into two categories, which are: (a) trigonometric method and (b) phase-locked loop (PLL) method [8]. The trigonometric method, also known as arctangent method, is simple and easy to implement. However, the trigonometric method only yields the unfiltered rotor position [9-12]. Usually, rotor velocity is obtained by a differential operation to rotor position. Nevertheless, the differential operation could amplify the noise in the resolver signals and cause large estimation error. A low-pass filter can be added to suppress the noise but at the expense of adding phase lag, which makes the trigonometric method lose its advantage of quick response.

The PLL method is a closed-loop strategy, can accurately track the rotor position and rotor velocity simultaneously, which makes it widely used in RDC ICs and software-based RDC. The estimates of rotor position and velocity are continuously corrected by the tracking loop. Most of RDC ICs adopt type II tracking loop as PLL structure, and this technique has been extended to software-based RDCs [13-16]. A PLL tracking algorithm on the basis of the type II tracking loop was proposed in [13] to achieve resolver-to-digital conversion with high precision. In [14], an RDC design using autotuning filters was presented to mitigate the resolver signal error on the speed output of the tracking loop. In [15], a novel and high-performance PLL-based resolver converter was proposed to measure angles in the full $360^{\circ}$ range. A PLL converter using PI controller was designed in [16] for resolvers and sine/cosine encoders. Besides, the angle tracking observer (ATO) methods presented in [17-19] are also PLL systems.

Both the type II tracking loop and ATO can track the rotor position and rotor velocity smoothly and accurately. Also, they have better performance in disturbance attenuation compared with the trigonometric method. However, the demodulation accuracy is still restricted by the system structure of conventional PLL methods. On the one hand, the estimation accuracies of type II PLL would be much lower when the rotor velocity varies quickly. This is because the type II system has inherent theoretical estimation errors. On the other hand, all the PLL-based RDC designs reviewed so far, suffer from the fact that there is a trade-off between dynamic performance and noise-suppression capability when designing the parameters of PLL. In other words, to quickly track the input and achieve better dynamic performance with little phase delay, the gain of PLL are usually set large to have wide closed-loop bandwidth, but high gain will make the PLL system extremely sensitive to the noise in resolver signals. Moreover, it may cause overshoot and instability if the parameters are not properly selected.

In this paper, a demodulation algorithm via Chebyshev filter-based Type III PLL is presented to estimate angular position and angular velocity from resolver signals. The proposed design is of a type III system, and it can obtain better filter characteristics without compromising dynamic performance, so as to achieve higher estimation precision in demodulation. Simulation and experiments are performed to verify the proposed method.

The rest of the paper is organized as follows. In Section 2, the principle of resolver and software-based RDC are introduced, and the problem of the paper is formulated. In Section 3, a Chebyshev filter-based type III PLL design is proposed for the demodulation of angular position and angular velocity, and the performance of the proposed method is analyzed by using Bode diagram. In Section 4, simulation and experiment are carried out to verify the effectiveness of the proposed demodulation method. Finally, conclusions are given in Section 5.

\section{Resolver Principles and Problem Formulation}

\subsection{Principle of Resolver and Software-Based RDC}

Resolvers are commonly used as shaft position sensing apparatus in servomotor control systems, and the schematic structure of a sine/cosine resolver is shown in Figure 1. It consists of one rotating winding (fixed on the rotor) and two stator winding. The rotor is directly installed on the motor 
shaft. If the rotating winding is supplied with a sinusoidal excitation signal $V_{e x}$, ideally, the two stator windings generate two orthogonal amplitude-modulated signals, which can be described as

$$
\left\{\begin{array}{l}
y_{\sin }=k E \sin \omega_{e} t \sin \theta \\
y_{\cos }=k E \sin \omega_{e} t \cos \theta
\end{array}\right.
$$

where $k$ is the transformation ratio of the resolver; $E$ and $\omega_{e}$ denote the amplitude and frequency of the excitation signal, respectively; $\theta$ is the rotor position of the resolver.

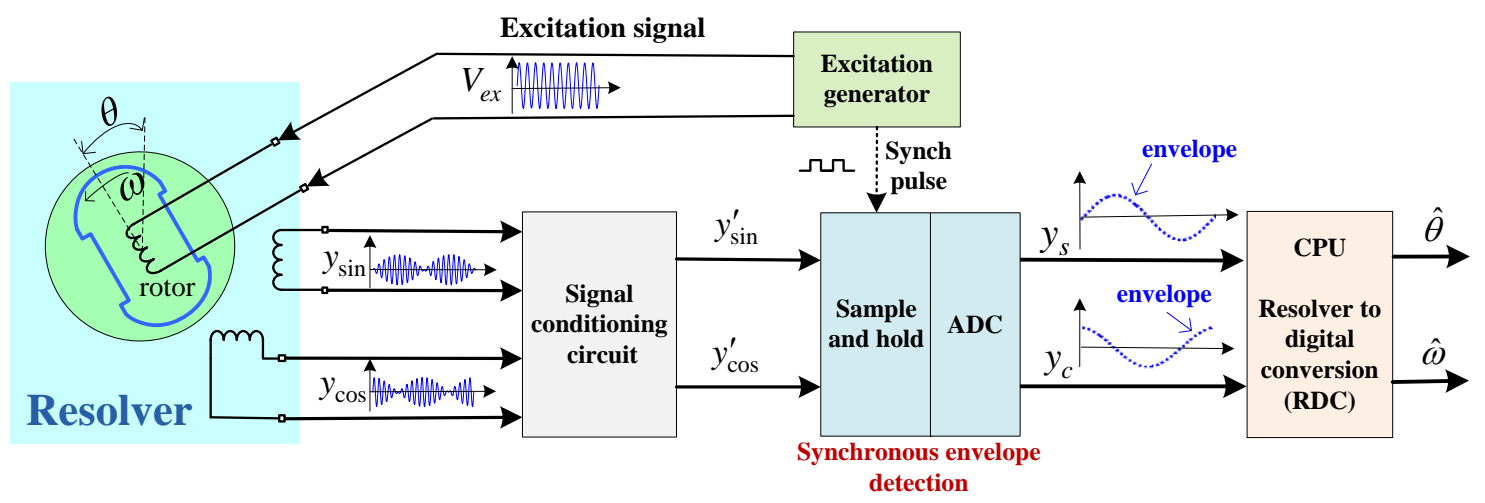

Figure 1. Schematic diagram of resolver and software-based RDC.

As shown in (1), the outputs of the resolver are two signals proportional to $\sin \theta$ and $\cos \theta$. Hence, to extract rotor position and velocity from the resolver output signals, further detection and demodulation are required. Detection refers to detecting the amplitude envelops from the resolver outputs; demodulation refers to the process of obtaining angular position and velocity from the envelop signals. Figure 1 illustrates the principle of software-based RDC. In order to relieve the pressure of microprocessor on sampling, the detection part is usually implemented by peripheral hardware circuits. After signal conditioning circuit, as in [20], the resolver signals are then synchronously demodulated by sampling at the peak of the sinusoid excitation signal in the sample and hold circuit. Next, the signals are sampled by A/D converters (ADC), which produces the digital value of sine and cosine envelope signals given by

$$
\left\{\begin{array}{l}
y_{s}=A \sin \theta \\
y_{c}=A \cos \theta
\end{array}\right.
$$

where $A$ is the amplitude of the detected envelope signals. Then, the envelope signals are demodulated by the software-based RDC algorithm to derive rotor position and velocity.

It should be noted that in practical applications, the resolver envelope signals are usually not ideal sine and cosine signals, where measurement errors and noise disturbances inevitably exist [21]. The resolver measurement errors (including amplitude deviation, DC offsets and phase shift) can be calibrated effectively [22]. Whereas, it is difficult to suppress the noise disturbance (including excitation signal interference and white noise in the circuits). Consequently, the actual resolver envelope signals can be written as

$$
\left\{\begin{array}{l}
y_{s}=A \sin \theta+n_{s} \\
y_{c}=A \cos \theta+n_{c}
\end{array}\right.
$$

where $n_{s}$ and $n_{c}$ are measurement noise. The influence of noise disturbance should be considered in the study on demodulation algorithm.

\subsection{Review of Conventional PLL-Based Demodulation Method}

The principle of PLL-based demodulation method is shown in Figure 2a. The PLL consists of three parts: a phase detector (PD), a loop filter (LPF), and a voltage-controlled oscillator (VCO). 
In software-based RDC, the VCO part can be modeled as an integrator. Define $\hat{\theta}$ and $\hat{\omega}$ as the estimates of angular position and velocity, and $\widetilde{\theta}=\theta-\hat{\theta}$ as the estimation error of angular position, then the output signal of PD can be given by

$$
\varepsilon=\sin \theta \cos \hat{\theta}-\cos \theta \sin \hat{\theta}=\sin (\theta-\hat{\theta})=\sin \widetilde{\theta}
$$

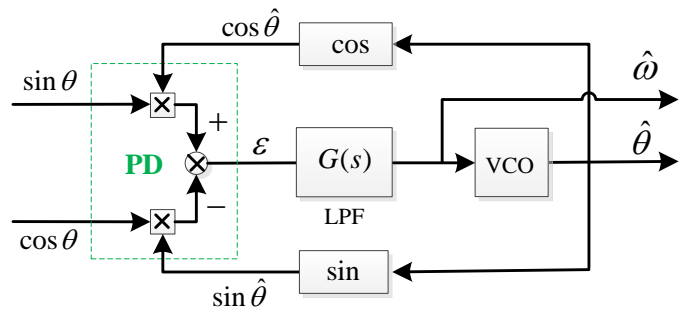

(a)

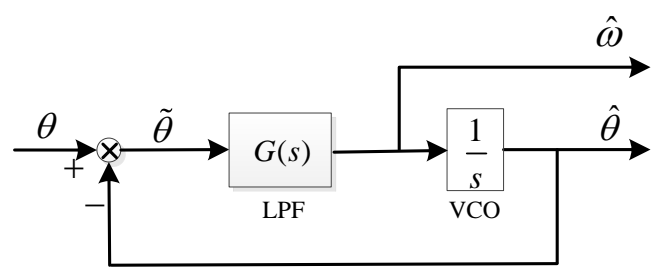

(b)

Figure 2. Block diagram of conventional PLL-based demodulation method. (a) Actual form; (b) simplified form.

When $\widetilde{\theta}$ is small enough, $\varepsilon=\sin \widetilde{\theta} \approx \widetilde{\theta}$. Based on this assumption, Figure 2a can be simplified as Figure 2 b. $G(s)$ plays an important role in the PLL structure of Figure 2, and it affects the performance of PLL in tracking angular position and angular velocity.

Most of RDC ICs adopt type II tracking loop as PLL structure, and it can be extended to software-based RDC. Taking the PLL structure of AD2S1210 [23] as an example, the simplified form of system response block diagram of AD2S1210 is shown in Figure 3.

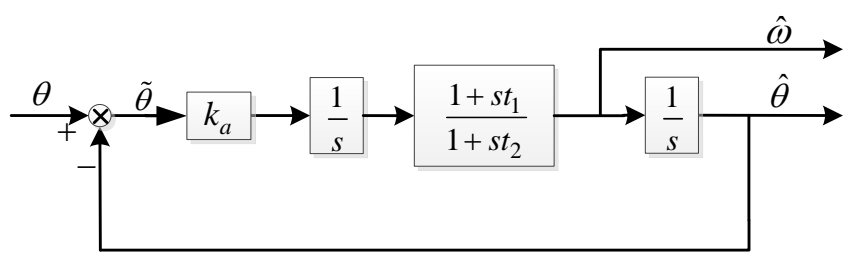

Figure 3. Simplified form of system response block diagram of AD2S1210.

According to Figure 3, the open loop transfer function of AD2S1210 can be described as

$$
\frac{G(s)}{s}=\frac{k_{a}}{s^{2}} \times \frac{1+s t_{1}}{1+s t_{2}}
$$

where $k_{a}>0$ is the open-loop gain, $1+s t_{1} / 1+s t_{2}$ is the compensation filter, and $t_{1}>t_{2}$ are the compensation time constants. Equation (5) shows that AD2S1210 is a Type II system. The transfer functions from $\theta(s)$ to $\hat{\theta}(s), \omega(s)$ to $\hat{\omega}(s)$ can be expressed as

$$
G_{\theta 1}(s)=\frac{\hat{\theta}(s)}{\theta(s)}=\frac{k_{a}\left(1+s t_{1}\right)}{t_{2} s^{3}+s^{2}+k_{a} t_{1} s+k_{a}}, G_{\omega 1}(s)=\frac{\hat{\omega}(s)}{\omega(s)}=\frac{k_{a}\left(1+s t_{1}\right)}{t_{2} s^{3}+s^{2}+k_{a} t_{1} s+k_{a}}
$$

The error transfer functions are

$$
E_{\theta 1}(s)=\frac{\widetilde{\theta}(s)}{\theta(s)}=\frac{t_{2} s^{3}+s^{2}}{t_{2} s^{3}+s^{2}+k_{a} t_{1} s+k_{a}}, E_{\omega 1}(s)=\frac{\widetilde{\omega}(s)}{\omega(s)}=\frac{t_{2} s^{3}+s^{2}}{t_{2} s^{3}+s^{2}+k_{a} t_{1} s+k_{a}}
$$

According to the final-value theorem, when the rotor runs at a constant velocity, AD2S1210 can achieve no-difference estimation of the rotor position and rotor velocity. When the rotor rotates at a constant acceleration of $A \mathrm{rad} / \mathrm{s}^{2}$, the steady state error of rotor velocity estimation is also equal to 0 , but for position estimate, there exists a certain tracking error which is equal to $A / k_{a}$. 
Equation (6) also indicates that AD2S1210 behaves like a third-order low-pass filter. At low frequencies, $s^{3}$ along with $s^{2}$ is very small and overwhelmed by the other terms, so (6) reduces to 1 , which means that the AD2S1210 generates so little of an effect that the rotor position and velocity can be accurately estimated. Whereas, at high frequencies, $s^{n}$ is very large, inducing attenuation and phase delay. Consequently, the gains of AD2S1210 are normally designed large enough to raise the effective bandwidth of RDC, which minimizes the phase delay, but this will make the system more sensitive to noise.

\section{Design of Chebyshev Filter-Based Type III PLL for Demodulation}

To improve the performance of PLL in demodulation algorithm, and meanwhile enhance its noise suppression ability, in this section, a Chebyshev filter-based type III PLL design is proposed.

\subsection{Design of Type III PLL}

Figure 4a shows the block diagram of Type III PLL design for the demodulation of angular position and angular velocity. $q_{1}, q_{2}, q_{3}$ are coefficients of the LPF in the PLL structure. The design of these coefficients will be explained in next part. As analyzed in Section 2.2, Figure 4a can be simplified as Figure 4b. In this demodulation method, the PLL is designed to be a type III system. Thus, the theoretical constant deviation problem of type II tracking loop in case of variable speed can be improved. Concrete analysis is as follows.
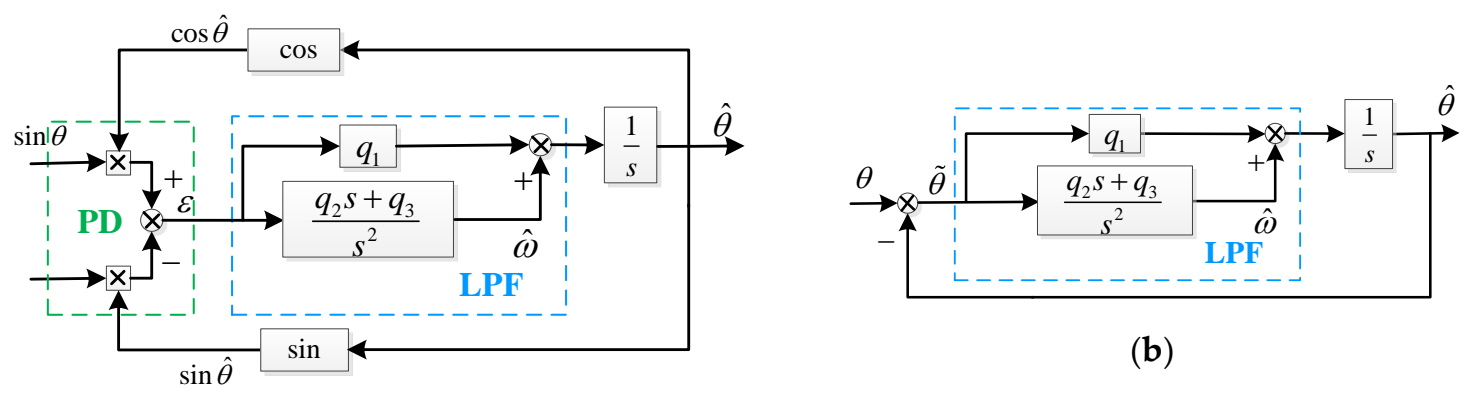

(a)

Figure 4. Block diagram of the Type III PLL design for demodulation. (a) Actual form; (b) Simplified form.

From Figure $4 \mathrm{~b}$, the transfer functions from $\theta(s)$ to $\hat{\theta}(s), \omega(s)$ to $\hat{\omega}(s)$ can be expressed as

$$
G_{\theta 2}(s)=\frac{\hat{\theta}(s)}{\theta(s)}=\frac{q_{1} s^{2}+q_{2} s+q_{3}}{s^{3}+q_{1} s^{2}+q_{2} s+q_{3}}, G_{\omega 2}(s)=\frac{\hat{\omega}(s)}{\omega(s)}=\frac{q_{2} s+q_{3}}{s^{3}+q_{1} s^{2}+q_{2} s+q_{3}}
$$

Then, the error transfer function can be written as

$$
E_{\theta 2}(s)=\frac{\widetilde{\theta}(s)}{\theta(s)}=\frac{s^{3}}{s^{3}+q_{1} s^{2}+q_{2} s+q_{3}}, E_{\omega 2}(s)=\frac{\widetilde{\omega}(s)}{\omega(s)}=\frac{s^{3}+q_{1} s^{2}}{s^{3}+q_{1} s^{2}+q_{2} s+q_{3}}
$$

According to the error transfer function in (9), when the rotor runs at a constant velocity, the proposed Chebyshev filter-based method can achieve no-difference estimation of the rotor position and rotor velocity. When the rotor rotates at a constant acceleration of $A \mathrm{rad} / \mathrm{s}^{2}$, the steady state error of rotor position estimate and velocity estimate are also equal to 0 .

\subsection{Parameter Design of Type III PLL via Chebyshev Filter}

After designing the type III PLL structure, the demodulation performance depends mainly on its parameters. As stated in precious section, in practical applications, there inevitably exist measurement noises in resolver signals. Seeing that, we need to carefully design the parameters of type III PLL to achieve the best estimation results. 
Equation (8) indicates that type III PLL is essentially equivalent to a low-pass filter, which filters out the high-frequency noise that may exist in the envelop signals. Therefore, in this paper, the problem of parameter design for type III PLL is transformed into a filter design problem. The design of low-pass filter should meet the following principles: (1) in the low frequency range, the frequency characteristics of filter should be as close as possible to 1 ; (2) while in the high frequency range, it should be as close to 0 as possible. That is, as close as possible to the characteristics of the ideal low-pass filter, thereby, not only can it obtain good estimation accuracy, but also effectively suppress high-frequency measurement noise.

\subsubsection{Introduction to Chebyshev Filter}

Here, we design the parameters of type III PLL on the basis of Chebyshev low-pass filter, whose characteristic is closer to the ideal low-pass filter [24]. The amplitude-frequency characteristic of the nth-order Chebyshev low-pass filter is as follows [25]

$$
|H(\omega)|=\sqrt{\frac{1}{1+\varepsilon^{2} T_{n}^{2}\left(\omega / \omega_{0}\right)}}
$$

where $\omega_{0}$ is the passband cut-off frequency, $T_{n}(\omega)$ is the Chebyshev polynomial, and $\varepsilon$ is the passband ripple factor. If the ripple factor is expressed in $\mathrm{dB}$, define

$$
\xi=-20 \lg \frac{1}{\sqrt{1+\varepsilon^{2}}}=10 \lg \left(1+\varepsilon^{2}\right)
$$

Then, the filter can be called a $\xi(\mathrm{dB})$ Chebyshev filter. For instance, $1 \mathrm{~dB}$ Chebyshev filter means that $\xi=1(\varepsilon=0.50885)$.

The amplitude-frequency characteristic of Chebyshev filter is determined by $\omega_{0}$ and $\xi$. $\omega_{0}$ can be selected properly according to the dynamic requirements of the system and the frequency range of the practical noise. As for $\xi$, its value affects the gain fluctuation and high-frequency amplitude characteristics in the passband of the filter. If the $\omega_{0}$ are set as $100 \mathrm{rad} / \mathrm{s}$, then, the bode diagram of the third-order Chebyshev filter with different $\xi$ is depicted in Figure 5. It shows that in the frequency range after $100 \mathrm{rad} / \mathrm{s}$, the larger the $\xi$, the smaller the high-frequency amplitude is, the better the noise suppression ability, but the fluctuation in the passband is intensified. Decreasing $\xi$ can reduce the amplitude fluctuation and phase angle lag in the passband, so as to get better dynamic property.

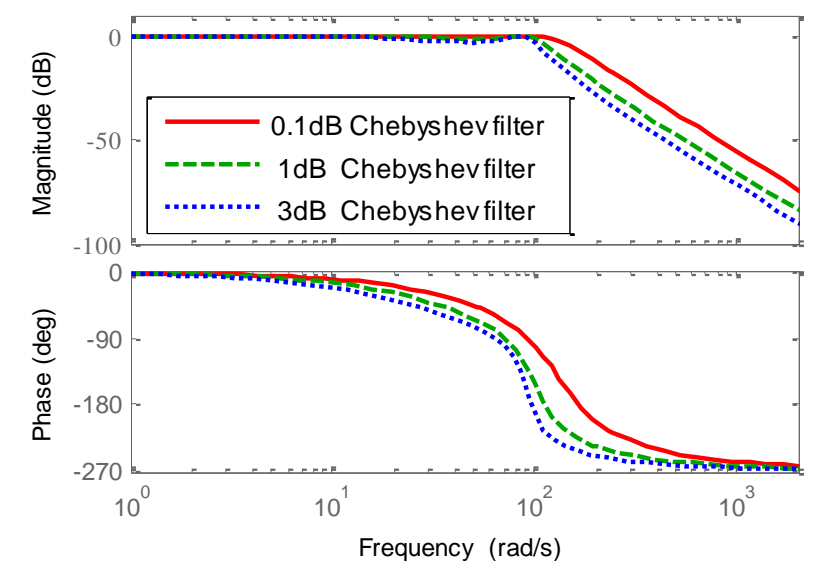

Figure 5. Bode diagram of the third-order Chebyshev filter with different $\zeta$.

After designing $\xi$, the transfer function of $n^{\text {th }}$-order Chebyshev filter can be expressed as

$$
H(s)=\frac{a_{n} \omega_{0}^{n}}{s^{n}+a_{1} \omega_{0} s^{n-1}+\cdots+a_{n-1} \omega_{0}^{n-1} s+a_{n} \omega_{0}^{n}}
$$


Take the third-order Chebyshev filter for instance, its transfer function is

$$
H(s)=\frac{a_{3} \omega_{0}^{3}}{s^{3}+a_{1} \omega_{0} s^{2}+a_{2} \omega_{0}^{2} s+a_{3} \omega_{0}^{3}}
$$

\subsubsection{Parameter Design of Type III PLL}

According to the coefficients of the third-order Chebyshev filter in (13), corresponding to (8), we can get the following parameters for type III PLL

$$
\left\{\begin{array}{l}
q_{1}=a_{1} \omega_{0} \\
q_{2}=a_{2} \omega_{0}^{2} \\
q_{3}=a_{3} \omega_{0}^{3}
\end{array}\right.
$$

In this way, the eigenvalues of type III PLL are placed to be the same position as those of a Chebyshev low-pass filter. Thus, demodulation parameters with better filter characteristics can be obtained. As analyzed in Section 3.2.1, the parameters can be easily tuned according to practical requirements and noise characteristics.

The coefficients of the third-order Chebyshev filter under different $\xi$ are listed in Table 1. Take 1 $\mathrm{dB}$ third-order Chebyshev filter $(\xi=1)$ for instance, the parameters of type III PLL can be set as

$$
\left\{\begin{array}{l}
q_{1}=0.98834 \omega_{0} \\
q_{2}=1.23841 \omega_{0}^{2} \\
q_{3}=0.49131 \omega_{0}^{3}
\end{array}\right.
$$

Table 1. Coefficients of the third-order Chebyshev filter.

\begin{tabular}{cccc}
\hline $\boldsymbol{\xi}(\mathbf{d B})$ & $\boldsymbol{a}_{1}$ & $\boldsymbol{a}_{2}$ & $\boldsymbol{a}_{3}$ \\
\hline 0.1 & 1.93881 & 2.62949 & 1.63805 \\
0.5 & 1.25291 & 1.53490 & 0.71569 \\
1 & 0.98834 & 1.23841 & 0.49131 \\
2 & 0.73782 & 1.02219 & 0.32689 \\
3 & 0.59724 & 0.92835 & 0.25059 \\
\hline
\end{tabular}

Therefore, after choosing $\xi$ there exists only one parameter $\omega_{0}$ that needs to be adjusted.

\subsection{Performance Analysis of the Proposed Method}

As for the speed servo system, to compare the performance of conventional type II tracking loop (AD2S1210) and the proposed Chebyshev filter-based type III PLL method, their parameters are set to make the closed-loop 3dB bandwidth of $G_{\omega}(s)$ identical when using the two methods. According to the parameters given in [23], the parameters of type II tracking loop are chosen as $k_{a}=46.3 \times 10^{3}$, $t_{1}=8 \times 10^{-3} s, t_{2}=728 \times 10^{-6} \mathrm{~s}$. Take $1 \mathrm{~dB}$ Chebyshev filter-based type III PLL for instance, to have the same $3 \mathrm{~dB}$ bandwidth, $\omega_{0}$ is set as $\omega_{0}=378 \mathrm{rad} / \mathrm{s}$. According to (15), the coefficients of the proposed method are $q_{1}=0.98834 \times 378, q_{2}=1.23841 \times 378^{2}, q_{3}=0.49131 \times 378^{3}$. Then, according to the transfer function in (6) and (8), the amplitude-frequency characteristics of angular position and velocity are illustrated in Figure 6 when using the two demodulation methods.

The transfer functions from $\theta(s)$ to $\hat{\theta}(s), \omega(s)$, to $\hat{\omega}(s)$ in (6) and (8) reflect the dynamic performance and noise suppression effect of the two demodulation methods. As depicted in Figure $6 \mathrm{~b}$, the closed-loop $3 \mathrm{~dB}$ bandwidth of $G_{\omega}(s)$ is identical $\left(\omega_{b w}=601 \mathrm{rad} / \mathrm{s}\right)$. It can be seen that compared with type II tracking loop, the curve of amplitude-frequency characteristic goes down in the high-frequency range by the proposed method, which indicates that the proposed method can suppress high-frequency noise more effectively in velocity estimates $\hat{\omega}$. While in Figure $6 \mathrm{a}$, for angular position 
estimates $\hat{\theta}$, the noise-suppression ability of the proposed method is worse but with larger bandwidth. Normally, the angular velocity estimates contain more noises than angular position estimates. Thus, the estimation accuracy of velocity would be improved greatly, and the angular position estimation results would be a little worse.

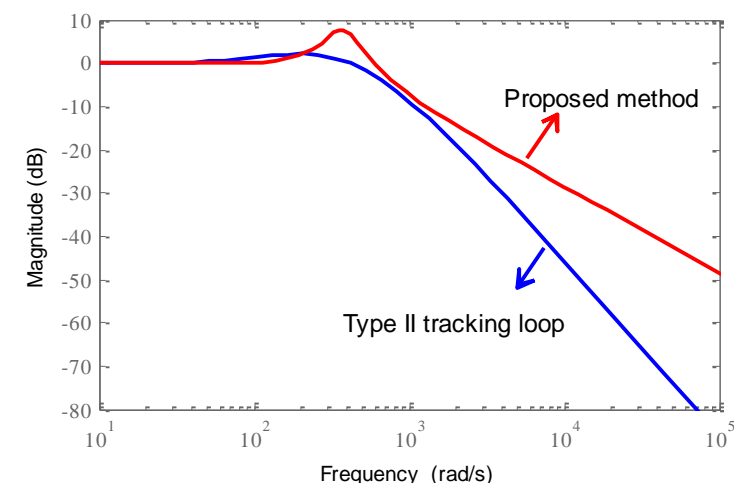

(a)

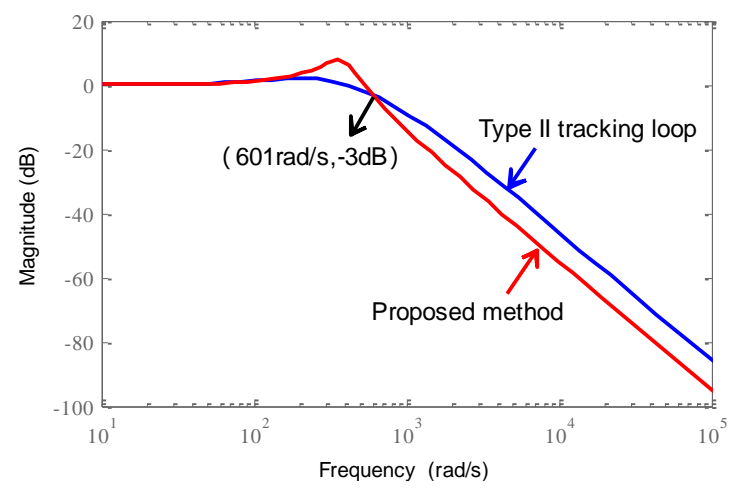

(b)

Figure 6. Amplitude-frequency characteristics of the two demodulation methods. (a) Angular position transfer function; (b) angular velocity transfer function.

Similarly, the amplitude-frequency characteristics of position and velocity error transfer function are depicted in Figure 7. As analyzed in [26], the error transfer functions from $\theta(s)$ to $\widetilde{\theta}(s), \omega(s)$ to $\widetilde{\omega}(s)$ in (7) and (9) show the ability in suppressing un-modeled dynamics. According to the results shown in Figure 7a,b, the proposed method has better performance in suppressing un-modeled dynamics.

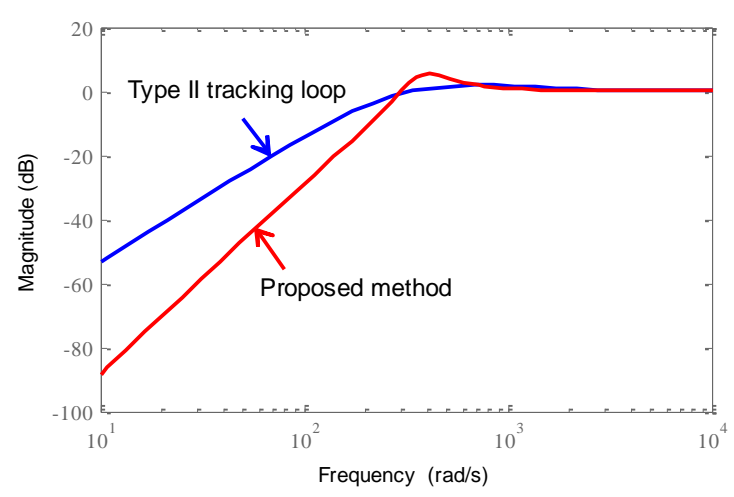

(a)

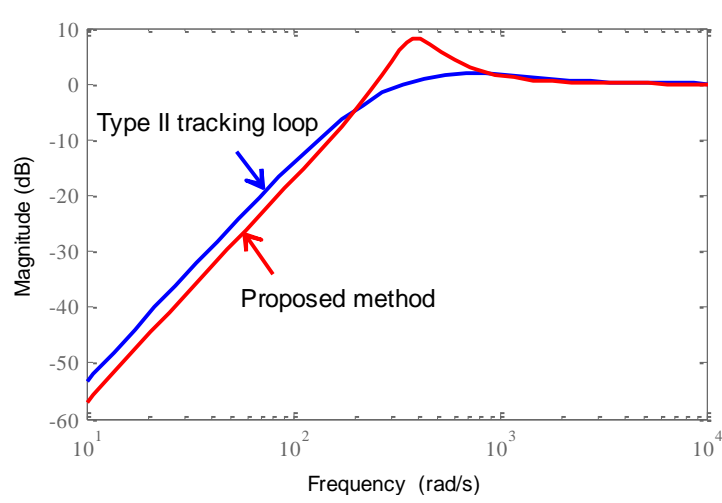

(b)

Figure 7. Amplitude-frequency characteristics of the two demodulation methods. (a) Angular position error transfer function; (b) angular velocity error transfer function.

From the above analysis, it can be seen that on the whole, the demodulation accuracy and noise-suppression ability can be improved by the proposed Chebyshev filter-based type III PLL method compared with the typical type II tracking loop method.

\section{Simulation and Experimental Results}

To verify the proposed demodulation algorithm for resolver signals, simulations and experiments are carried out.

\subsection{Simulation}

Figure 8 shows the semi-physical simulation platform. The resolver simulator takes a digital signal processor (DSP) TMS320F28335 (Texas Instruments Company, Dallas, TX, USA) as the core, and it can produce two envelope signals according to the preset value of angular position and velocity. 
Then, the envelope signals are converted into two analog signals $\left(y_{s}\right.$ and $y_{c}$ ) by Digital to Analog Converter (DAC). After that, the resolver envelopes are sampled by Analogl to Digital Converter (ADC) in the signal acquisition circuit, and then uploaded to the upper computer for the demodulation algorithm through the USB interface. The difference between the semi-physical simulation and the real motor experiment is that the actual angular position and angular velocity of the resolver are available, so that the performance of the two demodulation methods (type II tracking loop and the proposed Chebyshev filter-based type III PLL method) can be compared.

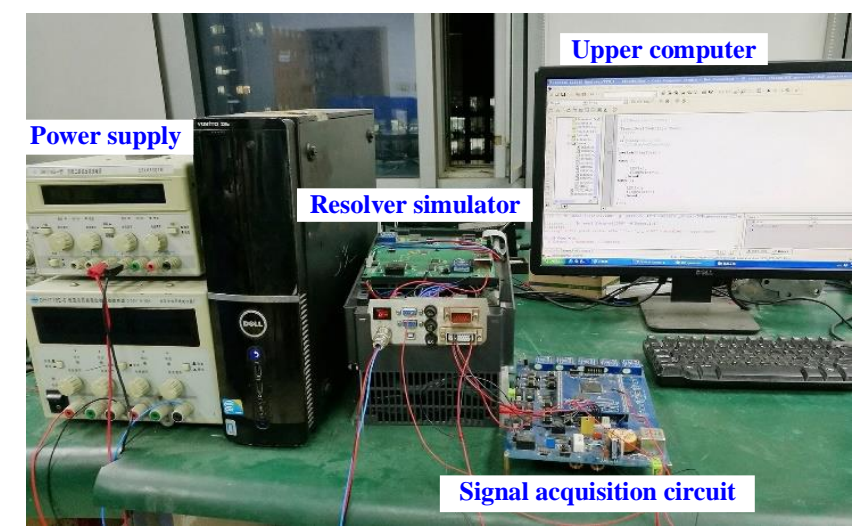

Figure 8. Semi-physical simulation platform.

As stated in previous section, demodulation parameters are set to make the closed-loop $3 \mathrm{~dB}$ bandwidth of $G_{\omega}(s)$-the transfer function of estimated velocity-identical when using the two methods. Take 1dB Chebyshev filter-based demodulation method for instance, the parameters are set as Section 3.3. The semi-physical simulation is carried out on the condition of two cases: constant speed $(2 \pi \mathrm{rad} / \mathrm{s})$ and constant acceleration $(10 \pi \cdot \mathrm{trad} / \mathrm{s})$.

\section{- Case 1: Constant Speed ( $2 \pi \mathrm{rad} / \mathrm{s})$}

In this case, the rotor position and velocity estimation errors of the two demodulation methods are presented in Figure 9. Meanwhile, the average (AVG) and standard deviation (STD) of estimation errors are given in Table 2 to evaluate the performance of the two demodulation methods.

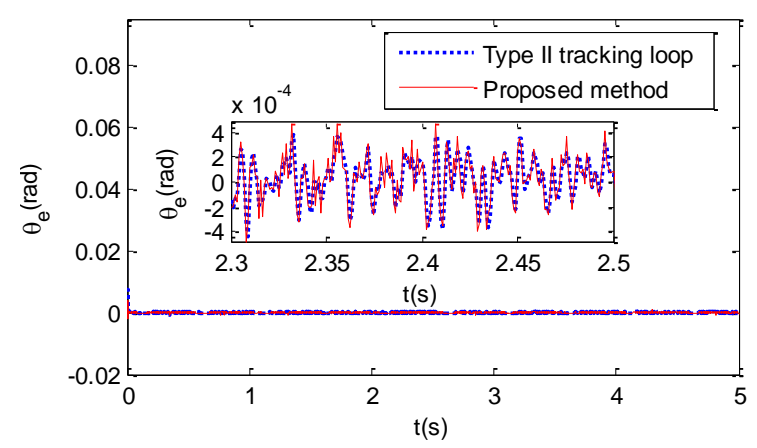

(a)

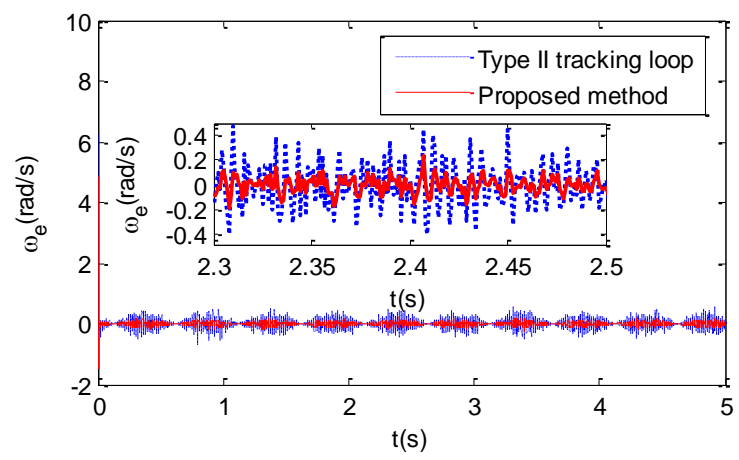

(b)

Figure 9. Estimation errors of the two demodulation methods in Case 1. (a) Rotor position estimation error; (b) rotor velocity estimation error.

As shown in Figure 9b, due to the closed-loop $3 \mathrm{~dB}$ bandwidths of $G_{\omega}(s)$ are identical, the convergence rates of velocity estimation error $\omega_{e}$ for the two demodulation methods are equal. In addition, from the enlarged views, we can see that the steady-state error curve of the proposed method is smoother compared with the type II tracking loop method. It means that the proposed method can attenuate the effects of measurement noise in resolver signals to some extent, thus, improve 
the steady-state accuracy of demodulation algorithm. While, for the estimation results of rotor position in Figure 9a, as analyzed in Section 3.3, the noise suppression capability is a little worse when using the proposed method, but its dynamic performance is improved.

The above analysis can also be proved by Table 2, from which we can see that the AVG of position and velocity estimation error is about the same, but the STD of velocity estimation error is reduced by $63 \%$ by the proposed method.

Table 2. Estimation error in simulation.

\begin{tabular}{lccccc}
\hline \multirow{2}{*}{ Cases } & \multirow{2}{*}{ Estimation Error } & \multicolumn{2}{c}{ Type II Tracking Loop } & \multicolumn{2}{c}{ Proposed Method } \\
\cline { 3 - 6 } & & AVG & STD & AVG & STD \\
\hline \multirow{2}{*}{ Case 1 } & Position estimation error (rad) & $1.951 \times 10^{-7}$ & $9.302 \times 10^{-5}$ & $1.877 \times 10^{-7}$ & $1.073 \times 10^{-4}$ \\
& Velocity estimation error (rad/s) & $-4.073 \times 10^{-5}$ & 0.0927 & $-2.112 \times 10^{-5}$ & 0.0340 \\
\hline \multirow{2}{*}{ Case 2 } & Position estimation error (rad) & $2.714 \times 10^{-4}$ & $9.416 \times 10^{-5}$ & $3.424 \times 10^{-7}$ & $1.085 \times 10^{-4}$ \\
& Velocity estimation error (rad/s) & $5.738 \times 10^{-5}$ & 0.0936 & $3.207 \times 10^{-5}$ & 0.0348 \\
\hline
\end{tabular}

- Case 2: Constant Acceleration $(10 \pi \cdot t \mathrm{rad} / \mathrm{s})$

In the case of constant acceleration, through the two demodulation methods, the rotor position and velocity estimation results are presented in Figure 10 and Table 2, which indicate that compared with the type II tracking loop method, the rotor position and velocity estimation errors are reduced by more than $60 \%$ when using the proposed method. As shown from the enlarged views in Figure 10b, the error curve of rotor velocity is smoother, which means that the proposed method can effectively suppress the high-frequency noise in the resolver signals. Therefore, the proposed method can achieve higher estimation precision than the type II tracking loop method. Moreover, it can be seen from Figure 10a and the AVG of estimation errors in Table 2 that, when motor runs at a constant acceleration, the proposed method can greatly reduce the theoretical constant deviation in the estimation results of type II tracking loop method (from $2.714 \times 10^{-4}$ to $3.424 \times 10^{-7}$ ). Therefore, in the case of variable speed, the proposed method can be adopted to accurately estimate rotor position and velocity.

From the above results, we may conclude that the proposed Chebyshev filter-based type III PLL method in this paper can achieve better performance no matter in the case of constant speed or the case of variable speed.

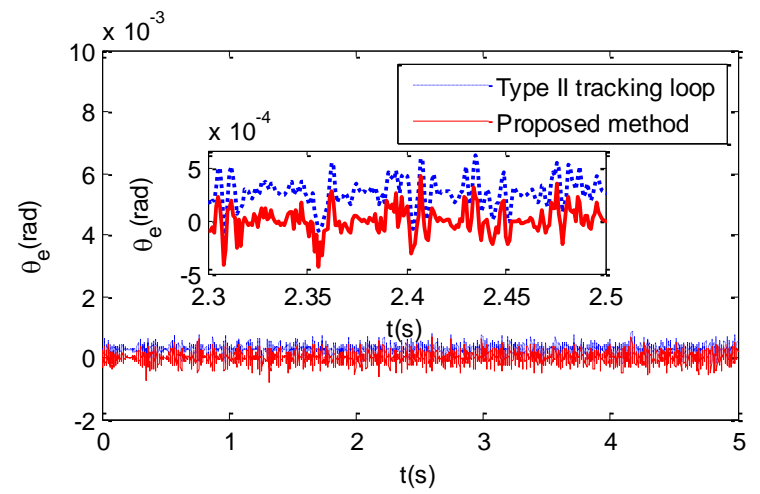

(a)

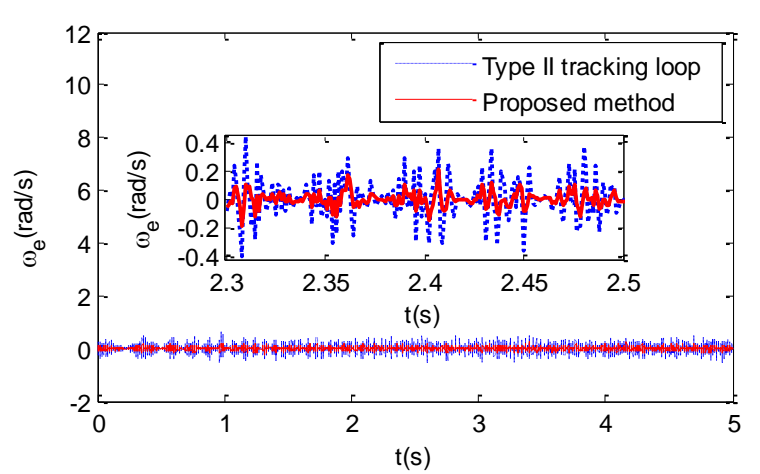

(b)

Figure 10. Estimation errors of the two demodulation methods in Case 2. (a) Rotor position estimation error; (b) rotor velocity estimation error.

\subsection{Experiment}

The experimental platform is illustrated in Figure 11, in which the PMSM (Infranor, Zurich, Switzerland) is equipped with a resolver. The parameters of PMSM and resolver are listed in Table 3. 
In the experiment, the drive and control board, which take DSP TMS320F28335 (Texas Instruments Company, Dallas, TX, USA) as the core, is used to drive the PMSM and implement signals processing unit for resolver outputs.

The PMSM is controlled to rotate at $\omega=2 \pi \mathrm{rad} / \mathrm{s}$ in the experiment. After signal conditioning and synchronous envelope detection circuits, the detected resolver envelopes are sampled by ADC, and then uploaded to the upper computer for demodulation algorithm through USB. The demodulation parameters are set as the simulation part. The derived rotor position and velocity are depicted in Figure 12a,b, respectively. Also, the AVG and STD of estimated velocity are computed to evaluate the performance of the proposed method as listed in Table 4 .

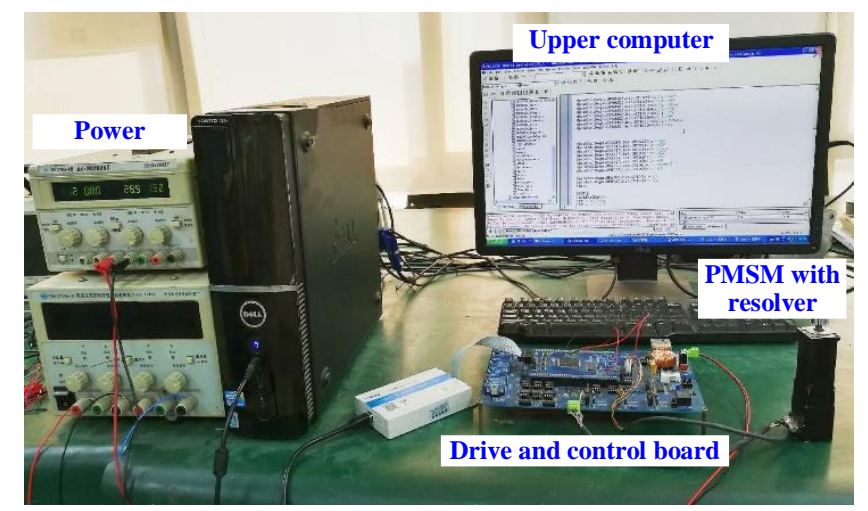

Figure 11. Experimental platform.

Table 3. PMSM and resolver parameters.

\begin{tabular}{cccc}
\hline \multicolumn{2}{c}{ PMSM } & \multicolumn{2}{c}{ Resolver } \\
\hline Pole pairs & 2 & Pole pairs & 1 \\
Rated voltage & $110 \mathrm{~V}(\mathrm{AC})$ & Input voltage & $5 \mathrm{~V} \pm 0.2 \mathrm{~V}(\mathrm{AC})$ \\
Rated speed & $3000 \mathrm{r} / \mathrm{min}$ & Input freguency & $10 \mathrm{kHz}$ \\
Torque constant & $0.15 \mathrm{Nm} / \mathrm{A}$ & Ouput voltage & $>2 \mathrm{~V}$ \\
Phase resistance & $8 \Omega$ & Transformer raio & $0.5 \pm 5 \%$ \\
Phase inductance & $10 \mathrm{mH}$ & Electrical error & $\leq 10^{\prime}$ \\
\hline
\end{tabular}

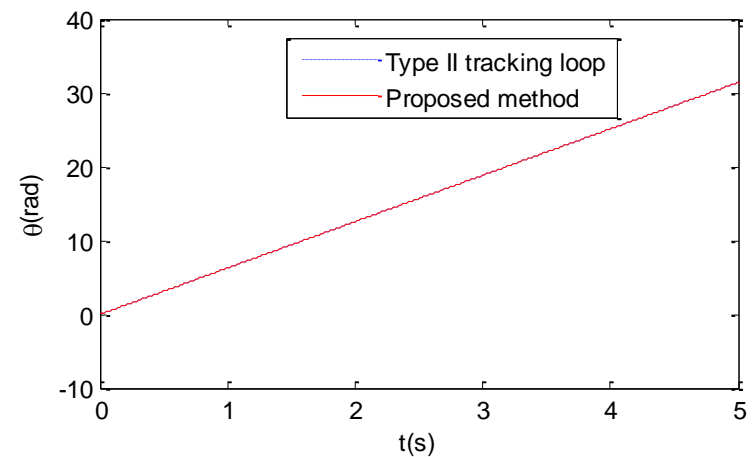

(a)

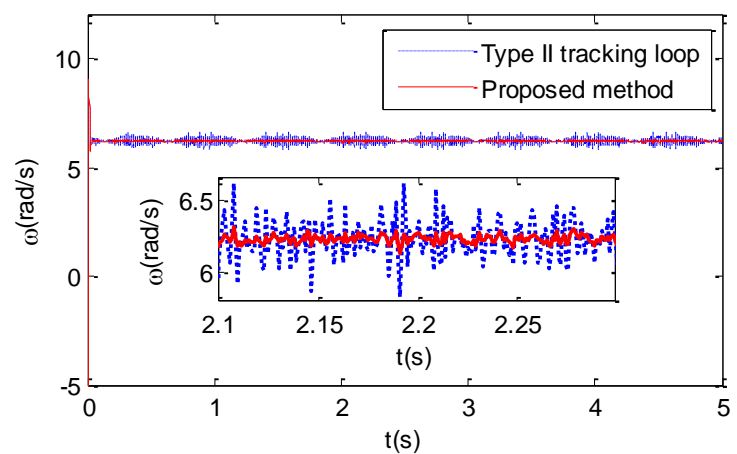

(b)

Figure 12. Estimated rotor position and velocity of the two demodulation methods. (a) Rotor position estimates; (b) rotor velocity estimates.

Table 4. Velocity estimates in experiment.

\begin{tabular}{ccc}
\hline Demodulation Methods & AVG & STD \\
\hline Type II tracking loop & 6.23 & 0.105 \\
Proposed method & 6.23 & 0.0319 \\
\hline
\end{tabular}


Note that the actual values of rotor position and velocity are unavailable in this physical experiment, so that the rotor position and velocity estimation errors cannot be obtained directly. The advantages of the proposed method can also be presented from the rotor velocity estimation results in Figure 12b. From the enlarged views in Figure 12b, it is obvious that the velocity estimation curve is smoother when using the proposed Chebyshev filter-based type III PLL method. It can also be seen from Table 4 that the STD of velocity estimates by the proposed method is smaller than that by the type II tracking loop (reduced by about 70\%). The proposed method is equally effective when the PMSM is working at other rates. Therefore, it can be concluded that compared with type II tracking loop, the proposed Chebyshev filter-based type III PLL method can improve the demodulation performance to a certain extent due to its stronger noise suppression capability.

\section{Conclusions}

In order to improve the angular position and velocity estimation accuracy of PLL-based demodulation method, this paper designs a Chebyshev filter-based type III PLL method for demodulation, which makes PLL become a system of type III, and meanwhile, the proposed method has stronger filter property to effectively suppress the high-frequency measurement noise in the resolver signals. Thus, the proposed method has higher demodulation accuracy compared with the conventional ones. Furthermore, the proposed Chebyshev filter-based parameter design method can also provide a theoretical guidance and reference for parameter selecting in other applications.

Author Contributions: H.L. and Z.W. designed the method and wrote the paper; H.L. performed the experiments and analyzed the data.

Funding: This work was partially supported by The National Natural Science Foundation of China (10772011).

Conflicts of Interest: The authors declare that there is no conflict of interests regarding the publication of this paper.

\section{References}

1. Han, S.; Han, S. Resolver angle estimation using parameter and state estimation. Measurement 2016, 93, 460-464. [CrossRef]

2. Benammar, M.; Ben-Brahim, L.; Alhamadi, M.A. A novel resolver-to- $360^{\circ}$ linearized converter. IEEE Sens. J. 2004, 4, 96-101. [CrossRef]

3. Caruso, M.; Di Tommaso, A.O.; Genduso, F.; Miceli, R.; Galluzzo, G.R. A DSP-Based Resolver-To-Digital Converter for High-Performance Electrical Drive Applications. IEEE Trans. Ind. Electron. 2016, 63, 4042-4051. [CrossRef]

4. Bergas-Jané, J.; Ferrater-Simón, C.; Gross, G.; Ramírez-Pisco, R.; Galceran-Arellano, S.; Rull-Duran, J. High-Accuracy All-Digital Resolver-to-Digital Conversion. IEEE Trans. Ind. Electron. 2012, 59, 326-333. [CrossRef]

5. Ellis, G. Encoders and Resolvers. In Control System Design Guide, 4th ed.; Butterworth-Heinemann: Boston, MA, USA, 2012; Volume 14, pp. 285-311.

6. Khaburi, D.A. Software-Based Resolver-to-Digital Converter for DSP-Based Drives Using an Improved Angle-Tracking Observer. IEEE Trans. Instrum. Meas. 2012, 61, 922-929. [CrossRef]

7. Guo, C.; Wu, C.; Ni, F.; Liu, H. Software-based resolver-to-digital conversion and online fault compensation. In Proceedings of the 2016 IEEE International Conference on Mechatronics and Automation, Harbin, China, 7-10 August 2016; pp. 344-349.

8. Sivappagari, C.M.R.; Konduru, N.R. Review of RDC soft computing techniques for accurate measurement of resolver rotor angle. Sens. Transducers 2013, 150, 1-11.

9. Sarma, S.; Agrawal, V.K.; Udupa, S. Software-based resolver-to-digital conversion using a dsp. IEEE Trans. Ind. Electron. 2008, 55, 371-379. [CrossRef]

10. Benammar, M.; Khattab, A.; Saleh, S.; Bensaali, F.; Touati, F. A Sinusoidal Encoder-to-Digital Converter Based on an Improved Tangent Method. IEEE Sens. J. 2017, 17, 5169-5179. [CrossRef] 
11. Pecly, L.; Schindeler, R.; Cleveland, D.; Hashtrudizaad, K. High-precision resolver-to-velocity converter. IEEE Trans. Instrum. Meas. 2017, 66, 2917-2928. [CrossRef]

12. Karabeyli, F.A.; Alkar, A.Z. Enhancing the accuracy for the open-loop resolver to digital converters. J. Electr. Eng. Technol. 2018, 13, 192-200.

13. Sun, J.D.; Cao, G.Z.; Huang, S.D.; Qiu, H. Software-based resolver-to-digital converter using the PLL tracking algorithm. In Proceedings of the International Conference on Ubiquitous Robots and Ambient Intelligence, Xi'an, China, 19-22 August 2016; pp. 719-723.

14. Qamar, N.A.; Hatziadoniu, C.J.; Wang, H. Speed Error Mitigation for a DSP-Based Resolver-to-Digital Converter Using Autotuning Filters. IEEE Trans. Ind. Electron. 2015, 62, 1134-1139. [CrossRef]

15. Benammar, M.; Gonzales, A.S.P. A Novel PLL Resolver Angle Position Indicator. IEEE Trans. Instrum. Meas. 2016, 65, 123-131. [CrossRef]

16. Alemadi, N.; Benbrahim, L.; Benammar, M. A new tracking technique for mechanical angle measurement. Measurement 2014, 54, 58-64. [CrossRef]

17. Harnefors, L.; Nee, H.P. A general algorithm for speed and position estimation of AC motors. IEEE Trans. Ind. Electron. 2000, 47, 77-83. [CrossRef]

18. Zhang, J.; Wu, Z. Composite state observer for resolver-to-digital conversion. Meas. Sci. Technol. 2017, 28, 065103. [CrossRef]

19. Raymundo, C.G.; João, O.P.P.; Suemitsu, W.I.; Soares, J.O. Improved demultiplexing algorithm for hardware simplification of sensored vector control through frequency-domain multiplexing. IEEE Trans. Ind. Electron. 2017, 64, 6538-6548.

20. Tiapkin, M.G.; Balkovoi, A.P. High resolution processing of position sensor with amplitude modulated signals of servo drive. In Proceedings of the 2017 IEEE Conference of Russian Young Researchers in Electrical and Electronic Engineering (EIConRus), St. Petersburg, Russia, 1-3 February 2017; pp. 1042-1047.

21. Alipour-Sarabi, R.; Nasiri-Gheidari, Z.; Tootoonchian, F.; Oraee, H. Performance Analysis of Concentrated Wound-Rotor Resolver for Its Applications in High Pole Number Permanent Magnet Motors. IEEE Sens. J. 2017, 17, 7877-7885. [CrossRef]

22. Wu, Z.; Li, Y. High-Accuracy Automatic Calibration of Resolver Signals via Two-Step Gradient Estimators. IEEE Sens. J. 2018, 18, 2883-2891. [CrossRef]

23. AD2S1210 Data Sheet: Variable Resolution, 10-Bit to 16-Bit R/D Converter with Reference Oscillator; Analog Devices, Inc.: Norwood, MA, USA, 2008; Available online: www.analog.com (accessed on 25 October 2018).

24. Kawakami, M. Nomographs for Butterworth and Chebyshev Filters. IEEE Trans. Circuit Theory 1963, 10, 288-289. [CrossRef]

25. Yahagi, T.; Wang, Y. Digital Filters and Signal Processing; The Science Publishing Company: Beijing, China, 2003; pp. 23-36.

26. Liu, H.; Wu, Z. On estimation algorithm of angular velocity for servo motors with resolvers. In Proceedings of the Chinese Control and Decision Conference, Shenyang, China, 9-11 June 2018; pp. 4019-4024. 